Revue

Revue de l'histoire des religions

de Ihistoire des religions

1 | 2014

Varia

\title{
Isabelle Poutrin, Convertir les musulmans. Espagne,
} 1491-1609

Paris, Presses Universitaires de France (« Le nœud gordien »), 2012

Alexandra Merle

\section{OpenEdition}

Journals

Electronic version

URL: http://journals.openedition.org/rhr/8225

DOl: $10.4000 /$ rhr.8225

ISSN: $2105-2573$

Publisher

Armand Colin

\section{Printed version}

Date of publication: 1 March 2014

Number of pages: 151-153

ISBN: 978-2200929107

ISSN: 0035-1423

Electronic reference

Alexandra Merle, «Isabelle Poutrin, Convertir les musulmans. Espagne, 1497-1609 », Revue de I'histoire des religions [Online], 1 | 2014, Online since 13 May 2014, connection on 22 September 2020. URL:

http://journals.openedition.org/rhr/8225 ; DOI : https://doi.org/10.4000/rhr.8225

This text was automatically generated on 22 September 2020 .

Tous droits réservés 


\title{
Isabelle Poutrin, Convertir les musulmans. Espagne, 1491-1609
}

Paris, Presses Universitaires de France (« Le nœud gordien »), 2012

\author{
Alexandra Merle
}

\section{REFERENCES}

Isabelle Poutrin, Convertir les musulmans. Espagne, 1491-1609, Paris, Presses Universitaires de France (« Le nœud gordien »), 2012, VI-363 p., $22 \mathrm{~cm}, 29,50 €$, ISBN

978-2-13-058914-3.

1 Les ambitions de cet ouvrage consacré aux morisques sont clairement énoncées dans l'introduction: ne pas se contenter d'être une utile synthèse en langue française des très nombreux travaux produits jusqu'à présent par les historiens espagnols mais aussi par certains hispanistes français - travaux considérablement augmentés ces derniers temps à l'occasion de la commémoration de l'expulsion de 1609 - sur l'histoire, les coutumes et l'identité des morisques, ces descendants des musulmans d'Espagne convertis à la foi chrétienne dans des circonstances telles que la sincérité de cette conversion pouvait être sujette à caution, et qui furent l'objet pendant des décennies de politiques d'assimilation et de répression et de débats animés avant que le constat d'échec de cette « acculturation » ne conduisît à la décision d'expulsion générale, sous Philippe III.

2 Isabelle Poutrin a souhaité aborder le sujet avec des outils qui n'ont pas toujours été suffisamment mis à profit auparavant, en croisant les sources ordinairement sollicitées (archives inquisitoriales, traités et réflexions sur les morisques, publiés ou manuscrits, chroniques, etc.) avec l'appareil juridique et théologique dont disposaient les autorités civiles et ecclésiastiques des $\mathrm{XVI}^{\mathrm{e}}$ et $\mathrm{XVII}^{\mathrm{e}}$ siècles, au moment des conversions et de l'expulsion, puisqu'il constitue le cadre théorique dans lequel ces mesures ont été prises. Outre le souci de restituer à ce cadre son importance afin d'éviter tout anachronisme - ce que n'ont pas toujours fait certains des travaux antérieurs qui ont 
recours aux notions de tolérance et d'intolérance en leur donnant un sens trop contemporain-, ce qui singularise l'ouvrage d'Isabelle Poutrin est la volonté de s'arrêter sur ces deux moments essentiels qui encadrent toute l'histoire des morisques, la conversion et l'expulsion, afin de mieux comprendre les raisons de ces mesures.

De cet objectif découle le plan de l'ouvrage, d'une grande simplicité apparente : une première partie est consacrée aux conversions (1491-1526), au pluriel car les musulmans devinrent des morisques d'abord dans le royaume de Castille, après la conquête de Grenade par les Rois Catholiques, dans la couronne d'Aragon ensuite, et dans des circonstances plus brutales liées à l'éclatement des Germanías de Valence sous Charles Quint. Une seconde partie intitulée « Le temps des doutes (1526-1609) » englobe toute la période qui va de la généralisation de la conversion dans la couronne d'Aragon à la date où fut décidée l'expulsion, longue période au sein de laquelle les spécialistes de l'histoire des morisques distinguent généralement des étapes : notamment avant et après la rébellion des morisques de l'ancien royaume de Grenade, dite "guerre de Grenade » ou « des Alpujarras » (1568-1570) qui, difficilement maittrisée par les troupes de Philippe II, se termina par le déplacement des populations morisques vers l'intérieur de la Castille dans le double but de les disperser et de les éloigner des côtes, pour prévenir toute entente avec les Barbaresques et les Turcs.

4 Tout au long de l'ouvrage, Isabelle Poutrin prend soin de replacer les mesures prises par les monarques dans un contexte qui dépasse le cadre géographique de la Péninsule. C'est ainsi qu'elle évoque les progrès des Turcs en Méditerranée et sur le continent européen au moment de la conquête de Grenade et montre que l'unification religieuse était inscrite dans la réalisation ultime de la Reconquête. L'analyse des capitulations accordées par les Rois Catholiques, si elle n'est pas entièrement neuve, met finement en valeur les omissions et les silences du texte, qui rendent possible une future conversion. Parmi les richesses de la première partie, on signalera aussi les nuances apportées à une opposition trop manichéenne entre les méthodes de Talavera, premier archevêque de Grenade, et celles de Cisneros, réputé aussi intransigeant que le premier était "tolérant", et la grande précision avec laquelle est traitée la conversion des musulmans de Valence, puis de ceux de toute la couronne d'Aragon. Afin de mieux comprendre la position de la commission réunie à Madrid pour statuer sur la validité des baptêmes réalisés pendant le soulèvement des Germanías puis la décision de généraliser la conversion, Isabelle Poutrin étudie minutieusement les écrits relatifs à cette question, notamment le Traité sur la conversion des païens de Fernando de Loazes (1525), et montre que ces réflexions s'appuient sur une longue tradition normative dont le poids ne saurait être négligé. C'est pour restituer à cette tradition toute son importance que le dernier chapitre de cette partie est consacré aux sources qui ont nourri les débats du début du $\mathrm{XVI}^{\mathrm{e}}$ siècle et permis de justifier les conversions, depuis le Décret de Gratien, le canon Maiores des Décrétales, et les écrits de Duns Scot, pour ne citer que les principaux textes mis à contribution.

5 L'analyse repose donc sur une mise en perspective double, par rapport à un contexte politique large (le statut de Charles Quint en tant qu'empereur et sa rivalité avec François $I^{\text {er}}$ ), et par rapport à une tradition juridique qui permet de valider des conversions forcées en employant la notion de «contrainte conditionnelle». La conclusion de cette analyse tend à minimiser la marge de manœuvre dont disposait Charles Quint en la circonstance, notamment vis-à-vis de l'Inquisition. Mais si celle-ci était alors, comme l'écrit Isabelle Poutrin, «l'un des instruments majeurs de l'autorité 
royale face aux nobles et aux villes et le rempart de l'Espagne contre les nouvelles doctrines venues d'Allemagne " (p. 169), il conviendrait sans doute de rappeler que son existence même fut menacée quelques années auparavant, lors de l'arrivée en Espagne de Charles et de ses conseillers flamands.

La même volonté de mise en perspective sous-tend la seconde partie de l'ouvrage, qui retrace les différentes phases de la réflexion sur les méthodes d'assimilation des morisques. Plusieurs points présentent un intérêt particulier : tout d'abord, Isabelle Poutrin montre toute la complexité de la réflexion sur les signes distinctifs des morisques, et au-delà sur leur assimilation au sein de la population «vieillechrétienne ", tantôt souhaitée (d'où les tentatives d'éradication de pratiques culturelles vues comme des obstacles à une conversion sincère), tantôt au contraire redoutée dans les moments où les morisques étaient perçus comme des ennemis de l'intérieur, qu'il était utile de pouvoir identifier aisément. Par ailleurs, elle met en évidence l'existence de voix discordantes et de jugements critiques sur les conditions de la conversion, très tôt dans le siècle, et sous des formes diverses, avant de s'intéresser aux progrès des recommandations visant à expulser les morisques, jusqu'à la prise de décision en 1609. Sur ce sujet qui a été très étudié, les apports de l'ouvrage sont réels: d'une part, Isabelle Poutrin parvient à nuancer l'opposition trop nette entre ceux qui prônent l'expulsion et ceux qui plaident encore pour une conversion authentique des morisques grâce à l'emploi de moyens variés. D'autre part, fidèle à la démarche suivie précédemment à propos de la conversion, elle replace la décision prise par Philippe III dans le temps présent du contexte international - soulignant après d'autres la relation qui existe entre la décision regardant les morisques et la nécessité où se trouvait la monarchie de restaurer un prestige fortement entamé en ces temps de trêve avec les Flamands - et surtout dans le temps long de l'histoire, ce qui permet d'engager une réflexion finale sur le caractère "exceptionnel » souvent prêté à l'expulsion des morisques.

$7 \mathrm{Au}$ total, cet ouvrage, qui manie une somme documentaire et bibliographique impressionnante et qui se distingue par la précision de ses analyses et par une écriture resserrée et d'une grande clarté, est bien à la hauteur de ses ambitions : il vient compléter la somme des études déjà consacrées aux morisques en rectifiant avec une rigueur non dépourvue d'élégance quelques idées reçues ou dérives auquel a pu prêter un tel sujet. Plus encore, il constitue une lecture indispensable pour quiconque s'intéresse aux politiques religieuses des monarchies modernes.

\section{AUTHORS}

\section{ALEXANDRA MERLE}

Université de Caen Basse-Normandie 